\title{
Low-energy electron collisions with sulfur hexafluoride, $\mathrm{SF}_{6}$
}

\author{
C. Winstead and V. McKoy \\ A. A. Noyes Laboratory of Chemical Physics, California Institute of Technology, Pasadena, California 91125
}

(Received 10 May 2004; accepted 14 June 2004)

We report calculated cross sections for elastic and electronically inelastic collisions of low-energy electrons with sulfur hexafluoride, $\mathrm{SF}_{6}$. Elastic cross sections are computed within the fixed-nuclei approximation, with polarization effects incorporated. Inelastic cross sections for nine low-lying states are computed in a few-channel approximation. We compare our cross sections to previous experimental and computational results where possible. () 2004 American Institute of Physics.

[DOI: $10.1063 / 1.1779214]$

\section{INTRODUCTION}

Sulfur hexafluoride, $\mathrm{SF}_{6}$, has long been important as a gaseous dielectric and more recently has found applications in plasma-based materials processing. ${ }^{1}$ Interactions of lowenergy (less than $\sim 50 \mathrm{eV}$ ) electrons with $\mathrm{SF}_{6}$ are thus of some practical interest and have received considerable study. ${ }^{1}$ However, the bulk of attention has been given to electronically elastic collisions at very low energies, in which attachment to form long-lived $\mathrm{SF}_{6}^{-}$metastables can occur, and to ionizing collisions. Comparatively few studies have been done of elastic scattering over a wide energy range. Of these, particularly noteworthy are the recent experimental studies by Cho and co-workers, ${ }^{2,3}$ who measured differential cross sections up to $75 \mathrm{eV}$ at scattering angles up to $180^{\circ}$, and the recent calculations of Gianturco and co-workers, ${ }^{4,5}$ who computed elastic cross sections up to 100 eV. Low-energy electron-impact excitation of $\mathrm{SF}_{6}$ has received still less attention, despite its critical importance to dissociation of $\mathrm{SF}_{6}$ in plasma applications; indeed, the only published measurements appear to be the 1977 results of Trajmar and Chutjian, ${ }^{6}$ who obtained energy-loss spectra as a function of angle at low impact energies, from which they extracted approximate differential cross sections at $20 \mathrm{eV}$.

We report here results from a study of elastic and inelastic collisions of low-energy electrons with $\mathrm{SF}_{6}$ using the Schwinger multichannel (SMC) method ${ }^{7,8}$ as implemented for parallel computers. ${ }^{9,10}$ The elastic integral, differential, and momentum-transfer cross sections were computed with polarization effects included, while inelastic cross sections were obtained within a few-channel approximation for the nine lowest-lying singlet and triplet excited states.

\section{COMPUTATIONAL DETAILS}

The SMC method and its implementation have been discussed in several publications. ${ }^{7-10}$ Here we give details specific to the present calculations.

All scattering calculations were carried out in the fixednuclei approximation, assuming $O_{h}$ symmetry and an S-F bond length of $1.56 \AA$. Because we neglect nuclear motion, the electronically elastic cross section we obtain does not distinguish between the vibrationally elastic and vibrationalexcitation cross sections. In $\mathrm{CF}_{4}$, for example, shape reso- nances in our computed elastic cross section ${ }^{11}$ were not seen in the measured vibrationally elastic cross section ${ }^{12,13}$ but were quite prominent in the vibrational-excitation ${ }^{12,14}$ and total-scattering ${ }^{15}$ cross sections. At very low impact energies, a fixed-nuclei description of $\mathrm{SF}_{6}$ scattering is certain to be qualitatively incorrect, because the dominant process (formation of metastable anions) involves nuclear motion.

Single-excitation configuration-interaction (SECI) calculations were carried out using GAUSSIAN, ${ }^{16}$ with the built-in 6-311+ $\mathrm{G}(d)$ basis set, to identify low-lying excited states. Based on those results, we chose to calculate excitation cross sections for the five lowest triplet states, namely, $1{ }^{3} T_{1 g}$, $1{ }^{3} T_{1 u}, 1{ }^{3} T_{2 u}, 1{ }^{3} T_{2 g}$, and $1{ }^{3} E_{g}$, and for each of the corresponding singlets except $1{ }^{1} E_{g}$, which was excluded because its SECI threshold $(15.23 \mathrm{eV})$ lay well above the $1{ }^{3} E_{g}$ threshold. All of the states considered here arise from promotion of a fluorine lone-pair electron into the lowest unoccupied molecular orbital (LUMO), which is the S-F antibonding $6 a_{1 g}$ orbital.

The ground state was described by a single-configuration restricted Hartree-Fock wave function. Single-configuration descriptions of the excited electronic states of $\mathrm{SF}_{6}$ were computed in the improved virtual orbital (IVO) approximation. ${ }^{17}$ As currently implemented, the SMC method requires that the same wave function be used to describe singlet and triplet states having the same orbital configuration. We used the triplet IVO wave functions in the present work; thresholds for the singlet channels were computed as expectation values of the singlet IVO Hamiltonian. GAMESS (Ref. 18) was used to generate the ground- and excited-state electronic wave functions.

As Hay ${ }^{19}$ found in an earlier study, $\mathrm{SF}_{6}$ thresholds determined by the IVO method exhibit unusually large errors. Comparison with the SECI results indicates that most but not all of the energy error is due to the single-configuration description of the excited state in the IVO approach. However, the SECI calculations do confirm that, for the states considered here, the wave functions are predominantly of ( $F$ lone-pair) $\rightarrow 6 a_{1 g}$ character. The thresholds obtained in the present calculations are collected in Table I along with experimental values. It should be noted that the assignment of the experimental thresholds remains controversial. In Table I we follow the assignments proposed by Trajmar and 


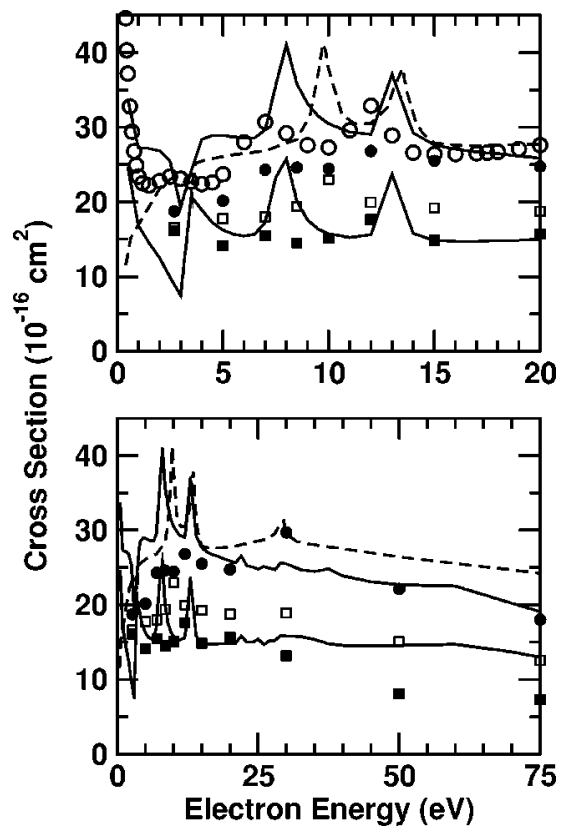

FIG. 1. Cross sections for elastic scattering of electrons by $\mathrm{SF}_{6}$. Integral cross sections shown are present results (upper solid curve), calculations of Gianturco and Lucchese (Ref. 5) (dashed curve), and measurements of Cho and co-workers (Refs. 2 and 3) (circles). Momentum-transfer cross sections are from present work (lower solid curve), Gianturco and Lucchese (open squares), and Cho and co-workers (filled squares). The upper panel shows an expanded view of the low-energy region and includes for comparison the recommended total cross section of Ref. 1 (open circles).

Chutjian ${ }^{6}$ and by Hay; ${ }^{19}$ alternative assignments may be found elsewhere. ${ }^{20}$

The basis set used for all of the scattering calculations was the $6-311+\mathrm{G}(2 d)$ basis set as defined in the program GAMESS (Ref. 18), which differs slightly from the basis set of the same name in GAUSSIAN (Ref. 16). All six Cartesian components of the $d$ functions were retained, giving a total of 211 contracted Cartesian Gaussian functions.

Preliminary elastic calculations were carried out in the static-exchange approximation, in which polarization effects
TABLE I. Thresholds for low-lying excited states of $\mathrm{SF}_{6}$.

\begin{tabular}{lcccr}
\hline \hline & \multirow{2}{*}{$\begin{array}{c}\text { Principal } \\
\text { character }\end{array}$} & \multicolumn{3}{c}{ Vertical threshold (eV) } \\
\cline { 3 - 5 } State & & SECI $^{\mathrm{a}}$ & IVO $^{\mathrm{b}}$ & Expt. $^{\mathrm{c}}$ \\
\hline $1{ }^{3} T_{1 g}$ & $1 t_{1 g} \rightarrow 6 a_{1 g}$ & 11.19 & 13.53 & \\
$1{ }^{3} T_{1 u}$ & $5 t_{1 u} \rightarrow 6 a_{1 g}$ & 11.78 & 14.43 & 11.6 \\
$1{ }^{1} T_{1 g}$ & $1 t_{1 g} \rightarrow 6 a_{1 g}$ & 12.17 & $13.88^{\mathrm{d}}$ & 9.8 \\
$1{ }^{3} T_{2 u}$ & $1 t_{2 u} \rightarrow 6 a_{1 g}$ & 12.18 & 14.85 & 11.0 \\
$1{ }^{3} E_{g}$ & $3 e_{g} \rightarrow 6 a_{1 g}$ & 12.25 & 14.72 & \\
$1{ }^{1} T_{2 u}$ & $1 t_{2 u} \rightarrow 6 a_{1 g}$ & 13.19 & $15.19^{\mathrm{d}}$ & 11.0 \\
$1{ }^{1} T_{1 u}$ & $5 t_{1 u} \rightarrow 6 a_{1 g}$ & 13.45 & $15.36^{\mathrm{d}}$ & 11.6 \\
$1{ }^{3} T_{2 g}$ & $1 t_{2 g} \rightarrow 6 a_{1 g}$ & 14.01 & 17.66 & 14.1 \\
$1{ }^{1} T_{2 g}$ & $1 t_{2 g} \rightarrow 6 a_{1 g}$ & 15.13 & $17.98^{\mathrm{d}}$ & 14.1 \\
\hline \hline
\end{tabular}

${ }^{\mathrm{a}} 6-311+\mathrm{G}(d)$ basis, GAUSSIAN (Ref. 16).

${ }^{\mathrm{b}} 6-311+\mathrm{G}(2 d)$ basis, GAMESS (Ref. 18).

${ }^{\mathrm{c}}$ Reference 6.

${ }^{\mathrm{d}}$ Computed with the $6 a_{1 g}$ orbital optimized for the triplet state having the same configuration.

are neglected. For the final elastic cross section, we included polarization effects for scattering wave functions belonging to the ${ }^{2} A_{1 g},{ }^{2} T_{1 u}$, and ${ }^{2} T_{2 g}$ symmetries, which are associated with low-energy shape resonances and which contain, respectively, $s, p$, and $d$ as their leading partial-wave contributions. Because it is easier to apply symmetry constraints in an Abelian group, polarized wave functions were actually constructed for the relevant representations of the $D_{2 h}$ subgroup, with the result that we also included some degree of polarization for ${ }^{2} E_{g},{ }^{2} T_{2 u}$, and ${ }^{2} T_{1 g}$; only for the very small ${ }^{2} E_{u}$ and ${ }^{2} A_{2 u}$ components was polarization completely neglected (the ${ }^{2} A_{1 u}$ component, also expected to be very small, is completely absent from our calculation). The additional, polarizing terms in the $(N+1)$-particle wave functions were constructed following a procedure ${ }^{21}$ in which a compact "resonance" orbital is kept occupied while allowing spin- and symmetry-preserving single excitations from the valence orbitals that describe relaxation of the target in the presence of the temporarily bound electron. For the $a_{1 g}, t_{1 u}$, and $t_{2 g}$ resonance orbitals, we used modified virtual

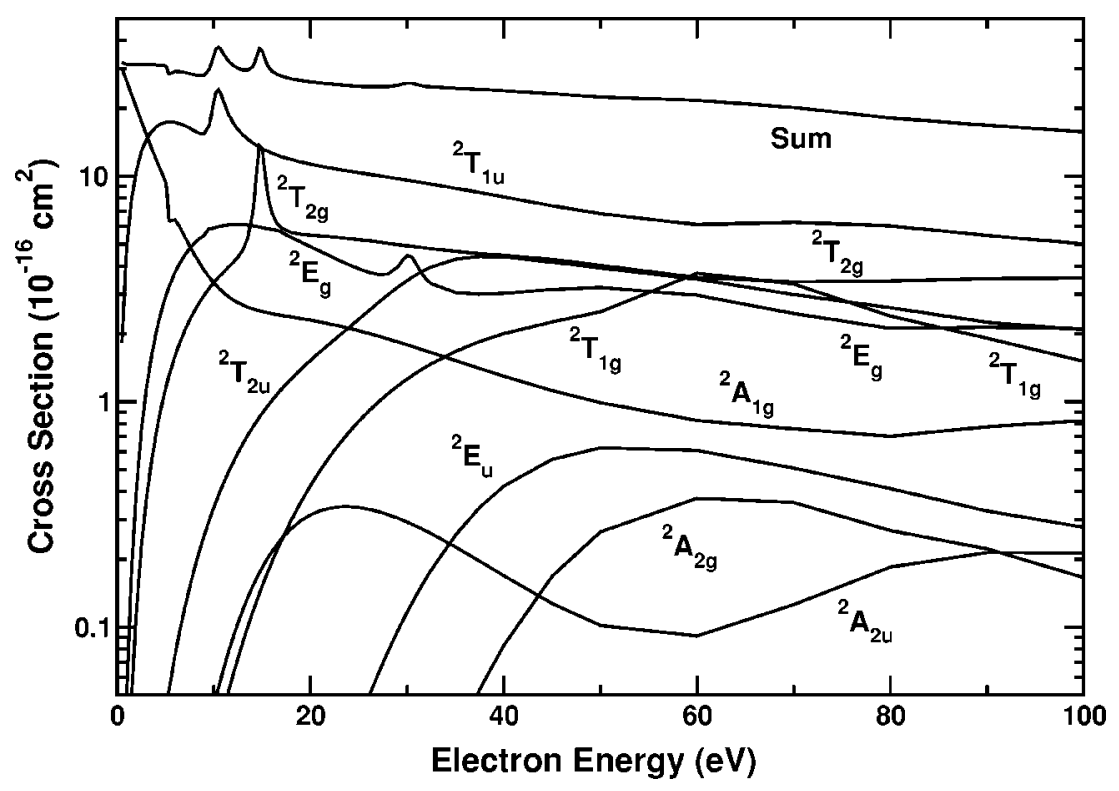

FIG. 2. $O_{h}$ symmetry components of the static-exchange integral elastic cross section. 

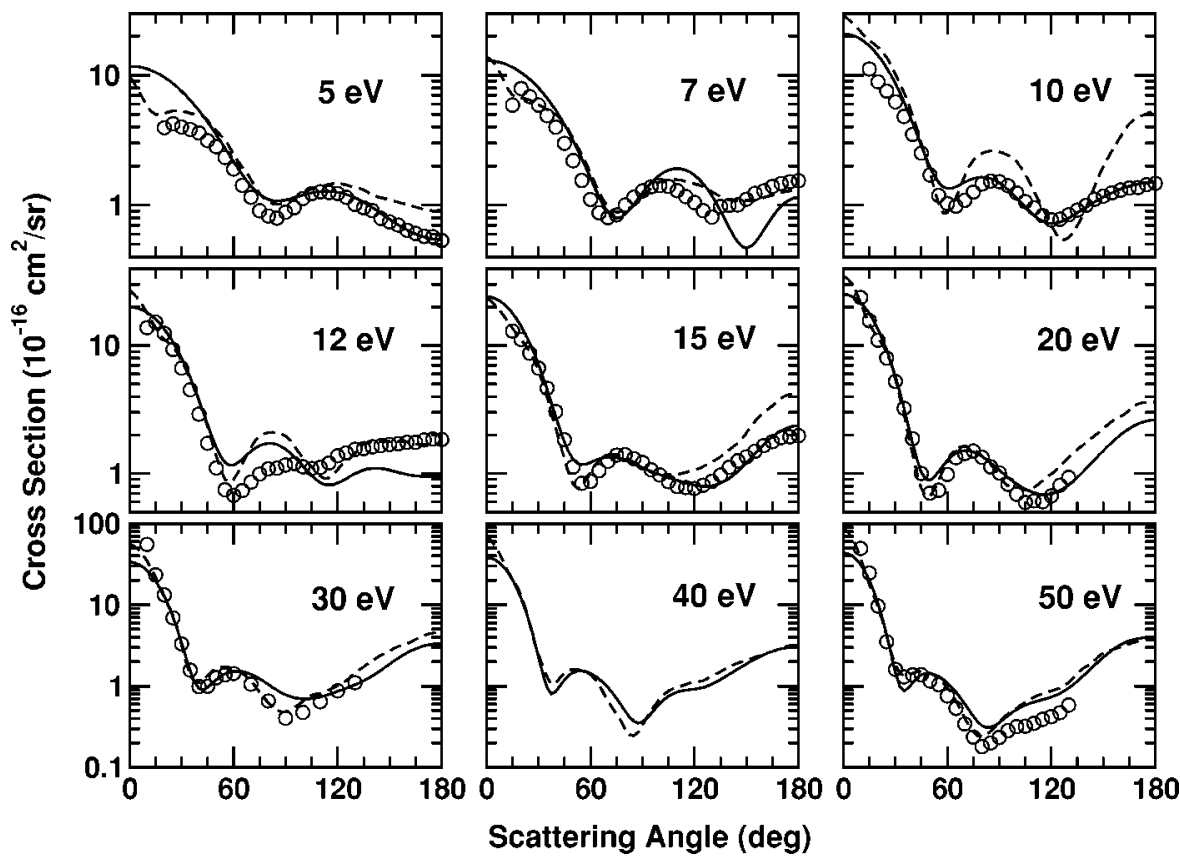

FIG. 3. Differential cross sections for elastic electron scattering by $\mathrm{SF}_{6}$ at selected energies. Results shown are present calculation (solid curves), calculations of Gianturco and Lucchese (Ref. 5) (dashes), and measurements of Cho and co-workers (Ref. 3) (circles).

orbitals $^{22}$ of the +6 cation. Because the $D_{2 h}{ }^{2} B_{n u}(n$ $=1-3$ ) components are equivalent for $\mathrm{SF}_{6}$, and likewise for ${ }^{2} B_{n g}$, only ${ }^{2} B_{3 u}$ and ${ }^{2} B_{2 g}$ scattering amplitudes were computed, with amplitudes for the other ${ }^{2} B_{n g, u}$ components obtained by rigid rotation.

Electronically inelastic cross sections were computed in a series of few-channel calculations. The $1{ }^{3} E_{g}$ cross section was obtained from a two-channel, three-state calculation (counting each degenerate component as a state) that included only $X^{1} A_{1 g}$ and $1^{3} E_{g}$. The remaining cross sections were obtained from three-channel, seven-state calculations, in which the triplet and singlet states having the same nominal configuration were coupled to each other and to the ground state. Of the excitations considered here, only that to the $1^{1} T_{1 u}$ state is optically allowed by dipole selection rules. In obtaining that cross section, we applied a correction for long-range scattering obtained from the dipole-Born approximation. $^{23}$ To compute the correction, we used a transition dipole, 0.7766 atomic units per component, computed for a ${ }^{1} T_{1 u}$ IVO state and retained partial waves up to ( $\ell$ $=5, m=5$ ) from the SMC calculation.

\section{RESULTS AND DISCUSSION}

\section{A. Elastic scattering}

The present integral elastic and momentum-transfer cross sections for electron scattering by $\mathrm{SF}_{6}$ are shown in Fig. 1. The upper panel shows the low-energy region in detail, while the lower panel covers a broader energy range. Also shown for comparison are recent computed ${ }^{5}$ and measured $^{2,3}$ results for the same cross sections. Figure 2 shows our static-exchange elastic cross section fully decom-

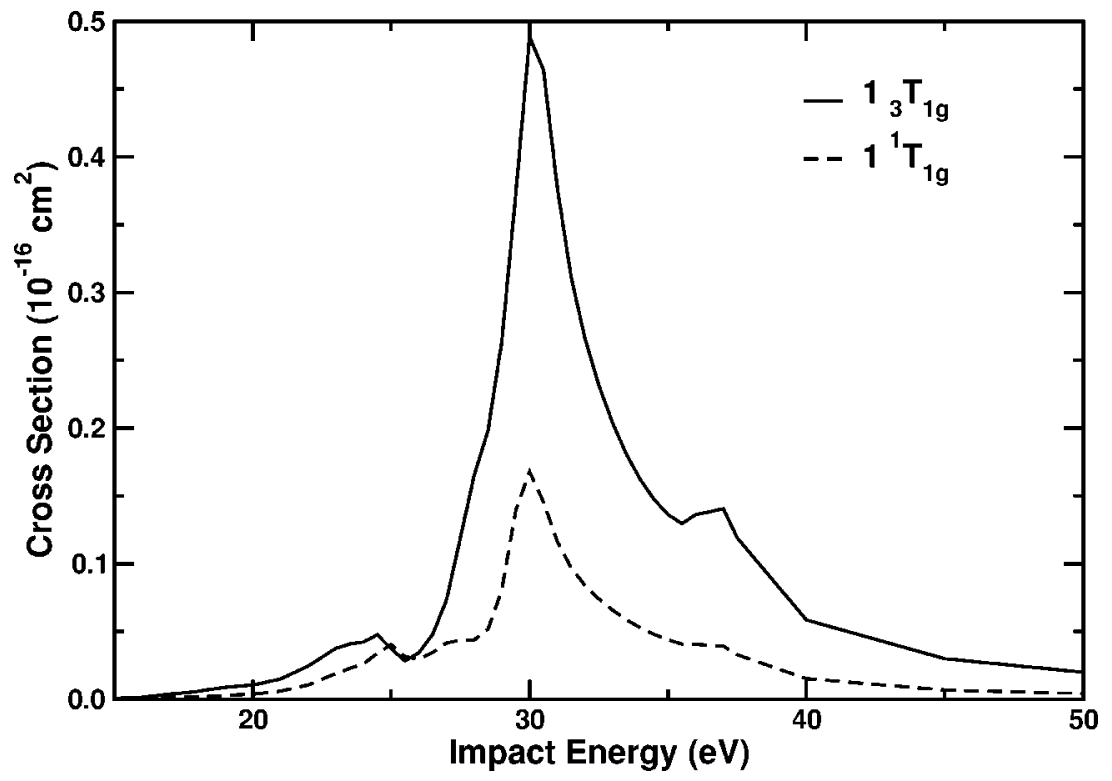

FIG. 4. Integral cross sections for electron-impact excitation of the ${ }^{1,3} T_{1 g}$ electronic states of $\mathrm{SF}_{6}$. 


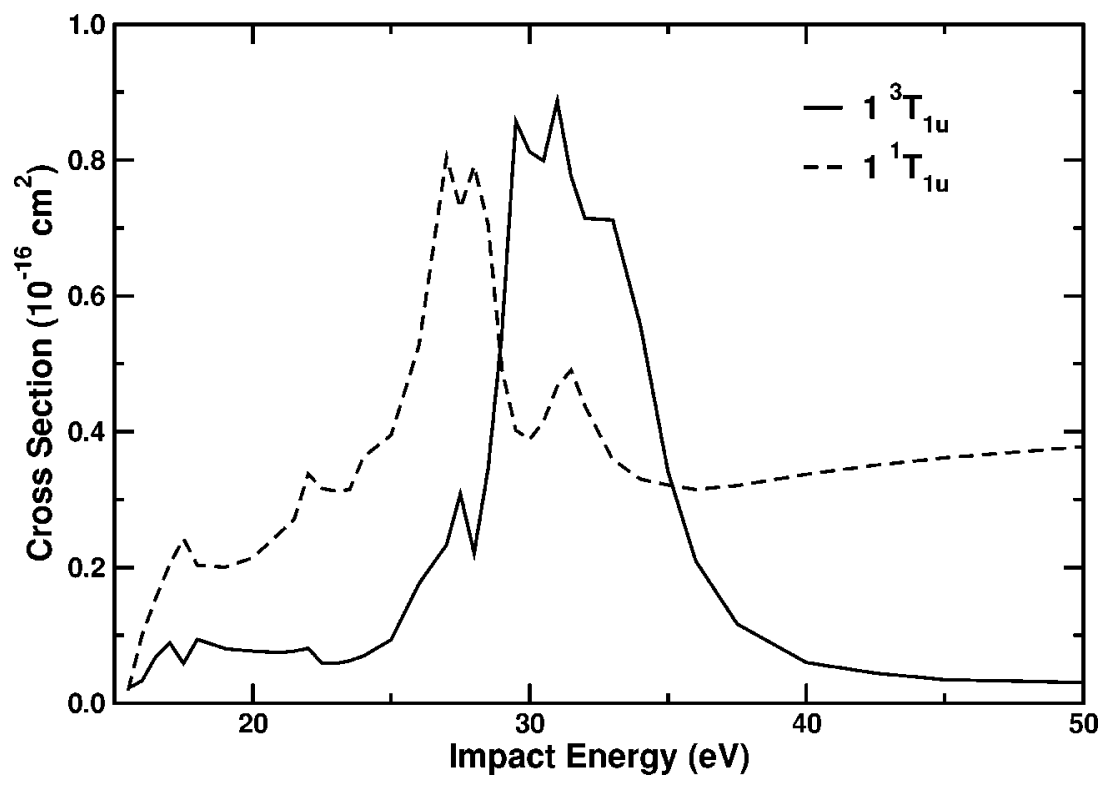

FIG. 5. Integral cross sections for electron-impact excitation of the $1^{1,3} T_{1 u}$ electronic states of $\mathrm{SF}_{6}$.

posed into $O_{h}$ components. Comparison with Fig. 1 confirms the identity of the prominent low-energy resonance features as ${ }^{2} A_{1 g},{ }^{2} T_{1 u}$, and ${ }^{2} T_{2 g}$ and gives an indication of the energy shifts of those features due to polarization. In Fig. 1, small oscillations visible from 20 to $40 \mathrm{eV}$ arise not only from the ${ }^{2} E_{g}$ resonance but also from pseudoresonances caused by treating as closed, in the representation of polarization, channels that are actually open at those energies; the ${ }^{2} E_{g}$ resonance is more clearly visible in the static-exchange results of Fig. 2.

At low energies, overall agreement with the calculation of Gianturco and Lucchese is generally good, although we predict slightly lower energies for the ${ }^{2} A_{1 g}$ and ${ }^{2} T_{1 u}$ resonances as well as a considerably greater width for the ${ }^{2} A_{1 g}$ feature. It is interesting to observe, however, that in both calculations the ${ }^{2} A_{1 g}$ resonance appears as a window rather than a peak in the fixed-nuclei cross section, in contrast to the earlier and more approximate calculations of Dehmer and Siegel $^{24}$ (not shown) and to the previous calculation by Gianturco and co-workers. ${ }^{4}$

The total electron scattering cross section, ${ }^{1}$ shown for comparison in the upper panel of Fig. 1, exhibits maxima at $2.5 \mathrm{eV}, 7 \mathrm{eV}$, and $12 \mathrm{eV}$. The energies we obtain for the ${ }^{2} A_{1 g}$, ${ }^{2} T_{1 u}$, and ${ }^{2} T_{2 g}$ resonances may be seen to agree reasonably well with the locations of these maxima. As mentioned above, fixed-nuclei "elastic" resonances do not necessarily appear in the vibrationally elastic cross section; indeed, although there is no indication of these resonances in the measured elastic cross sections shown in Fig. 1, measurements of vibrational excitation ${ }^{1}$ indicate that both the $T_{1 u}$ and the $T_{2 g}$ resonance contribute strongly to vibrational excitation.

At energies of $\sim 20 \mathrm{eV}$ and above, it may be observed from Fig. 1 that the present integral elastic cross section is in good agreement with the experimental values of Cho and

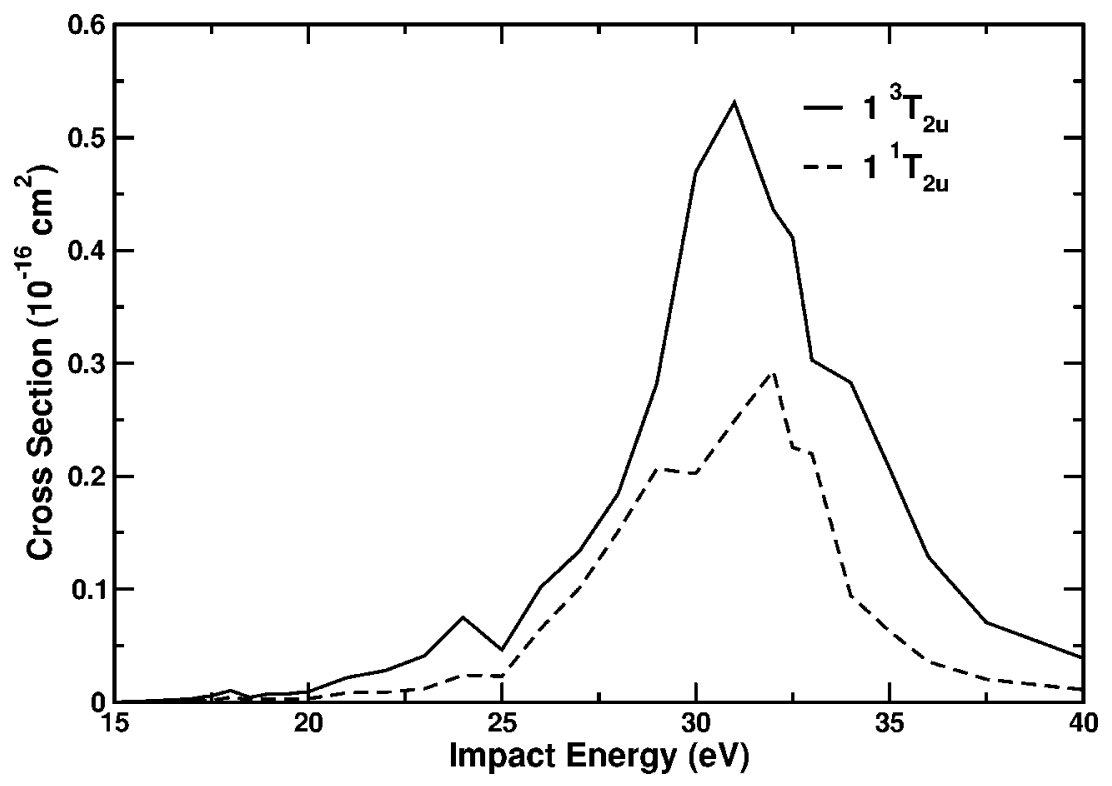

FIG. 6. Integral cross sections for electron-impact excitation of the $1^{1,3} T_{2 u}$ electronic states of $\mathrm{SF}_{6}$. 


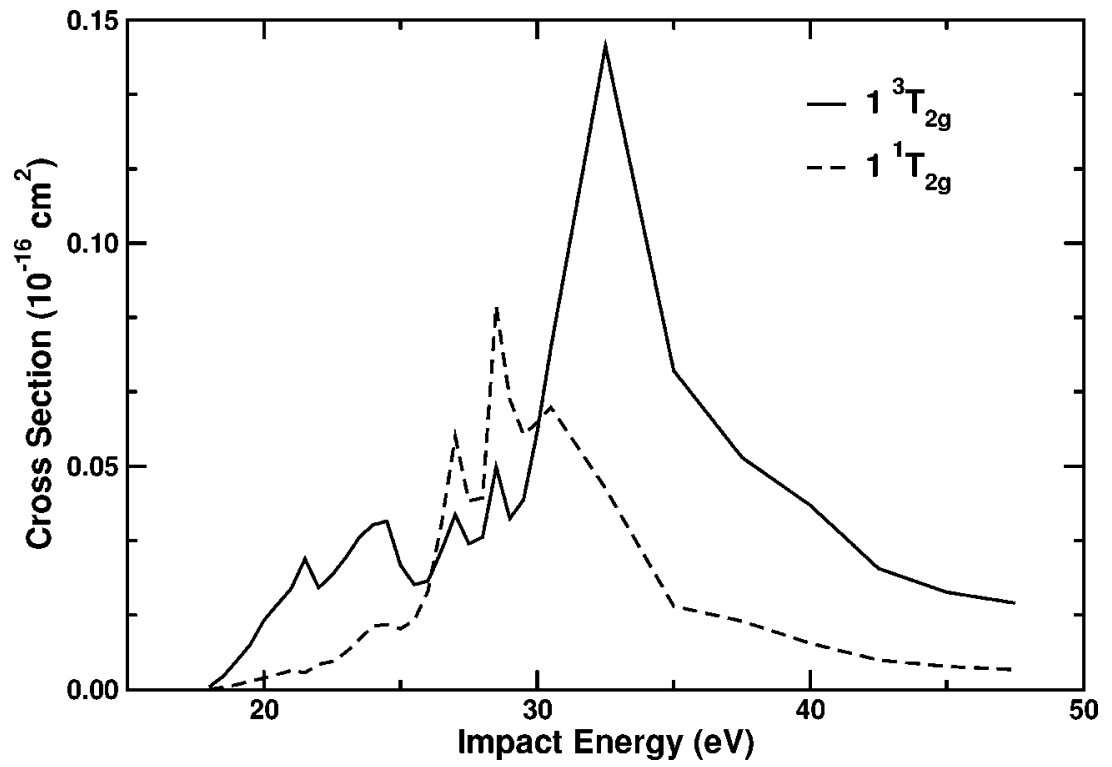

FIG. 7. Integral cross sections for electron-impact excitation of the $1^{1,3} T_{2 g}$ electronic states of $\mathrm{SF}_{6}$.

co-workers, ${ }^{2,3}$ except at $30 \mathrm{eV}$, and is significantly smaller than the theoretical values of Gianturco and Lucchese. ${ }^{5}$ The reasons for these discrepancies are unclear. Though we have checked that the quadrature we used to integrate over scattering angles remains converged at these higher energies, limitations in our one-particle basis set (particularly in its representation of components corresponding to high partial waves) can also lead to the computed cross section being too small at high energies. On the other hand, the total scattering and ionization cross sections appear to be well established in this energy range, on the basis of multiple experiments, ${ }^{1,25}$ and their difference places an upper limit on the elastic cross section that, while generally consistent with the present results, would seem to preclude values as large as those obtained by Gianturco and Lucchese (as well as the value reported by Cho and co-workers at $30 \mathrm{eV}$ ). For the momentum-transfer cross sections, the situation is reversed: above $20 \mathrm{eV}$ our results agree in magnitude with those of
Gianturco and Lucchese but not with those of Cho and coworkers, which fall below the calculations.

Differential elastic cross sections are shown at selected energies in Fig. 3. At very low energies, our results do not agree with the measured values, indicating the importance of a better treatment of polarization and/or consideration of nuclear-motion effects. However, from $\sim 5 \mathrm{eV}$ to $\sim 20 \mathrm{eV}$, agreement is quite good. The largest discrepancies are generally found in the near-forward direction, except at 7 and 12 $\mathrm{eV}$, where our calculated cross section appears to reflect stronger resonant influences than seen experimentally. At the highest energies, $50 \mathrm{eV}$ and above, both calculations predict more high-angle scattering than is measured, consistent with the difference in momentum-transfer cross sections (Fig. 1).

\section{B. Inelastic scattering}

Integral cross sections for electron-impact excitation of the nine states considered in the present study are shown in

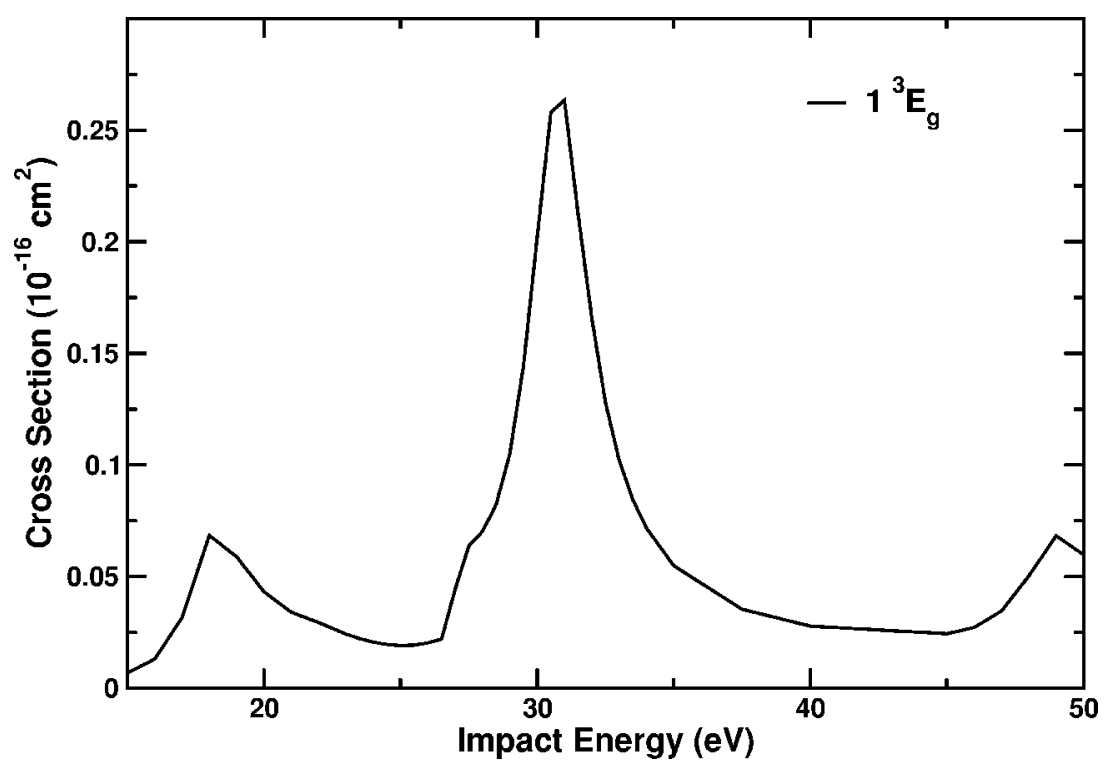

FIG. 8. Integral cross section for electron-impact excitation of the $1_{g}^{E}$ electronic state of $\mathrm{SF}_{6}$. 


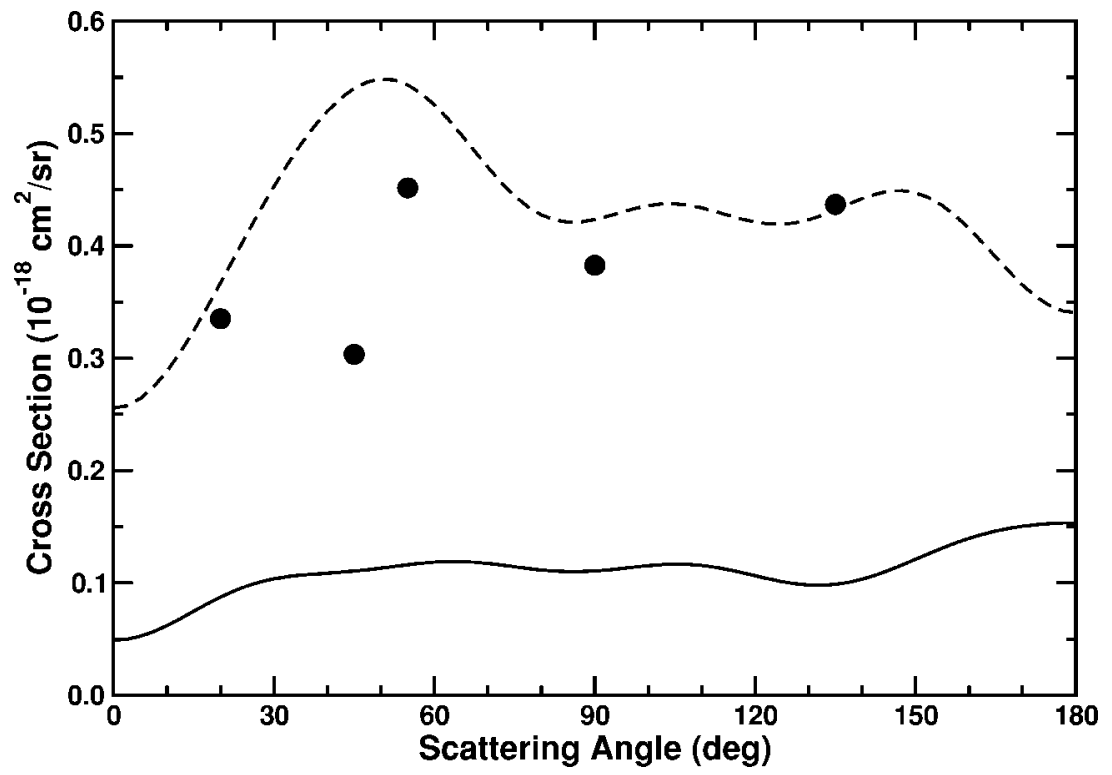

FIG. 9. Summed differential cross sections for electron-impact excitation of the $1{ }^{1,3} T_{1 g}$ states of $\mathrm{SF}_{6}$ at 20 $\mathrm{eV}$ (solid) and $23 \mathrm{eV}$ (dashed) impact energy, compared to measurements at $20 \mathrm{eV}$ (circles) by Trajmar and coworkers (Ref. 6).

Figs. 4-8. Although they differ considerably in magnitude, most of these cross sections are qualitatively similar, apart from small fluctuations that are probably numerical artifacts: they remain small from threshold to about $25 \mathrm{eV}$, then show broad peaks centered near $30 \mathrm{eV}$. The major exception is the cross section for the only optically allowed excitation we studied, $X \rightarrow 1{ }^{1} T_{1 u}$, which rises rapidly from threshold, displays a peak at about $28 \mathrm{eV}$, and remains large at higher energies. The cross section for excitation of the corresponding triplet state, $1^{3} T_{1 u}$, is also large, though smaller than the singlet cross section in the near-threshold and high-energy regions.

The origin of the broad peaks near $30 \mathrm{eV}$ in most of the excitation cross sections is unclear. One possible source of such peaks is core-excited shape resonances; however, another possibility is pseudoresonances associated with excitation and/or ionization channels that are treated as closed in the present calculations but are, in fact, open. Calculations employing more extensive coupling schemes would be useful in assessing the latter possibility. Under the circumstances, we can at least say that our calculations suggest that in the low-energy, near-threshold region, which is of primary importance in modeling of low-temperature plasmas, the $1^{1,3} T_{1 u}$ excitations appear to be the most important among those studied here.

As noted in the Introduction, experimental data on the electron-impact excitation cross sections of $\mathrm{SF}_{6}$ are extremely limited, but they do include differential cross sections for five energy-loss processes measured at $20 \mathrm{eV}$ impact energy. ${ }^{6}$ If we accept the assignments of Trajmar and co-workers for those processes, we can compare our results to theirs for three cases, with results shown in Figs. 9-11. From Fig. 9, we see that agreement is fair if we compare the measured result for the $9.8 \mathrm{eV}$ energy-loss process to our summed singlet and triplet $T_{1 g}$ cross sections at $20 \mathrm{eV}$ impact energy: both experiment and calculation indicate the

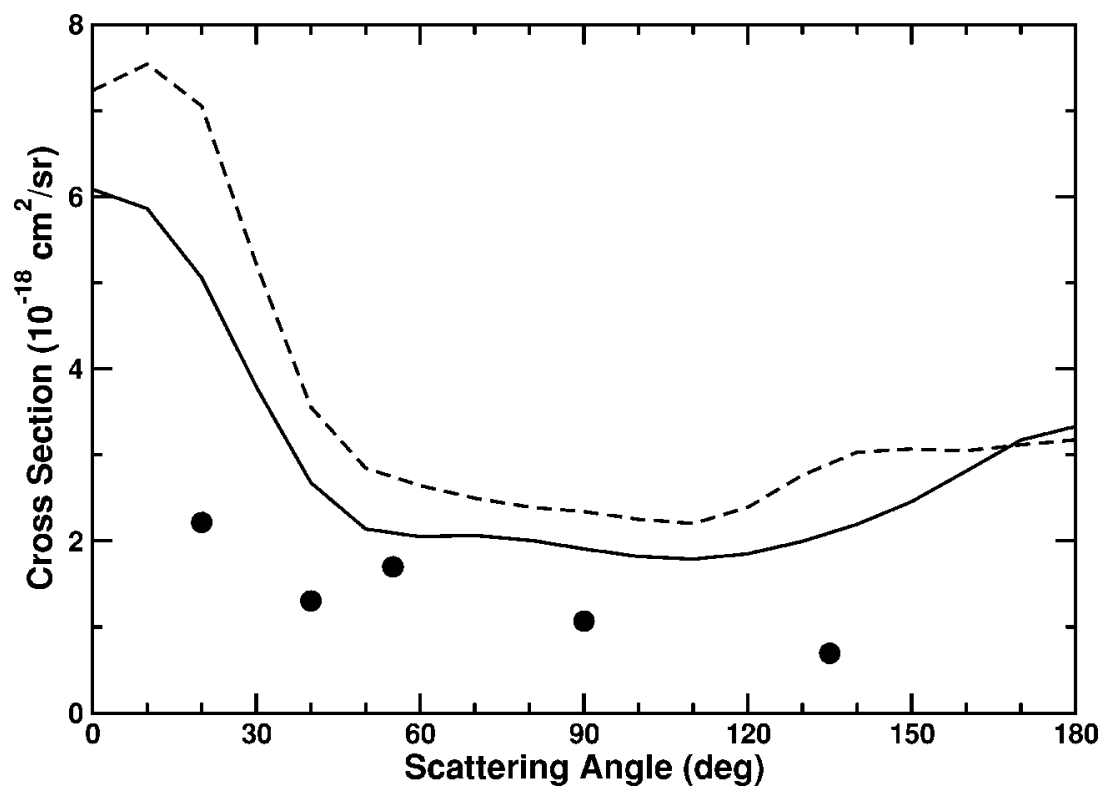

FIG. 10. As in Fig. 9, for $1{ }^{1,3} T_{1 u}$. 


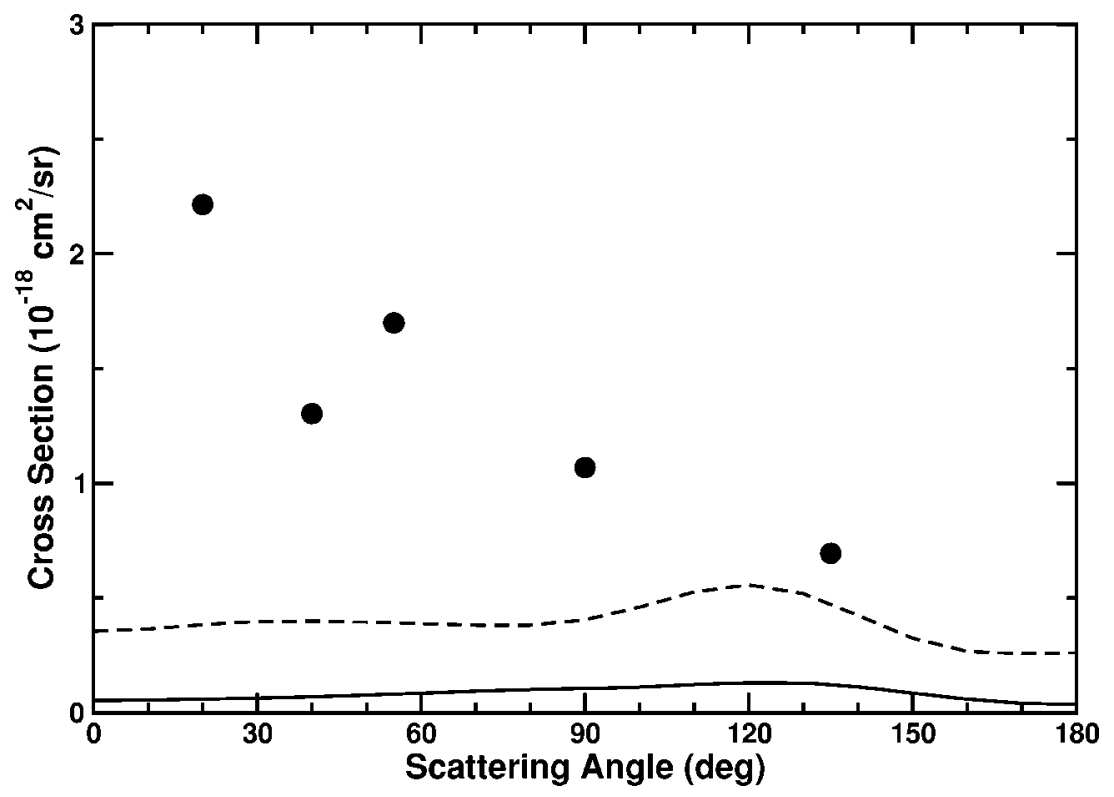

FIG. 11. As in Fig. 9, for $1{ }^{1,3} T_{2 u}$.

cross section is quite small and fairly isotropic. Indeed, if we allow for the error in the computed threshold by comparing instead to results calculated at $23 \mathrm{eV}$ impact energy, so that the residual energy is approximately the same as in the experiment, agreement is rather good.

For the $11.6 \mathrm{eV}$ process that Trajmar and co-workers assign as $1^{1,3} T_{1 u}$, agreement is at best qualitative (Fig. 10). Although calculation and experiment both produce forwardpeaked cross sections that are of the same order of magnitude and much larger than those for $1^{1,3} T_{1 g}$ shown in Fig. 9, the differences between theory and experiment are considerable. In particular, the computed cross section is larger and more forward-peaked than the measured cross section.

The remaining case where it is possible to compare is excitation of the $1^{1,3} T_{2 u}$ states, which Trajmar and coworkers assign to the energy-loss process at $11.0 \mathrm{eV}$ and for which they report the cross section to be the same, within experimental error, as for the $11.6 \mathrm{eV}$ energy-loss process assigned as ${ }^{1,3} T_{1 u}$. As seen in Fig. 11, where we show our summed $1^{1,3} T_{2 u}$ cross sections plotted against the same experimental points as in Fig. 10 (note the difference in vertical scale between the figures), there is no agreement between calculation and measurement. Though it appears reasonable to us that the summed $1{ }^{1,3} T_{2 u}$ cross section should, as our calculations indicate, be more similar to the $1^{1,3} T_{1 g}$ cross section of Fig. 9 than to the $1^{1,3} T_{1 u}$ cross sections of Fig. 10, since only the ${ }^{1} T_{1 u}$ transition is optically allowed, we certainly cannot rule out the larger cross section indicated by the experiment.

Trajmar and co-workers also report cross sections at 20 $\mathrm{eV}$ for excitation processes with thresholds at 12.8 and 13.3 $\mathrm{eV}$, which, following Hay, ${ }^{19}$ they assign as $1 t_{1 g} \rightarrow 7 a_{1 \mathrm{~g}}$ and $1 t_{1 g} \rightarrow 6 t_{1 u}$. The former cross section is the same, within experimental error, as those for the 11.0 and $11.6 \mathrm{eV}$ processes, while the latter is similar in shape but larger. Although neither cross section is particularly large in an absolute sense, both are surprisingly large in comparison to those of lower-lying states. Because we focused on the lowest sev- eral singlet and triplet states as indicated by the SECI calculations, all of which involved excitation into the $6 a_{1 \mathrm{~g}}$ LUMO, we did not compute cross sections for these processes. When using the present results as a starting point to model near-threshold excitation of $\mathrm{SF}_{6}$, neglect of such additional channels must be borne in mind.

\section{SUMMARY}

We have calculated elastic and electronic-excitation cross sections for low-energy electron collisions with $\mathrm{SF}_{6}$. Our elastic cross sections are in generally good agreement with available experimental results in the energy region where the approximations made in the calculation are valid, and they are also in good agreement with previous calculations except at the higher energies studied, where the present results appear more consistent with the measured total and ionization cross sections.

Our electron-impact excitation cross sections are in at best fair agreement with experimental data; however, those data are very limited, and both the calculation and experiment involve large uncertainties. Further studies of electronimpact excitation of this important molecule, both experimental and theoretical, would therefore be desirable. Nonetheless, the present results can, together with other data available in the literature, contribute to construction of an electron cross section set for $\mathrm{SF}_{6}$ in which the limitations and uncertainties of the input data are in part compensated for by validating predictions based on the set against electron swarm measurements, ${ }^{26}$ with systematic adjustment of the cross sections as necessary to achieve agreement with the macroscopic properties. Construction of such a cross section set will be reported separately. ${ }^{27}$

\section{ACKNOWLEDGMENTS}

This work was supported by the U.S. Department of Energy, Office of Basic Energy Sciences. The authors thank W. Lowell Morgan for helpful discussions. We acknowledge In- 
tel Corporation for an equipment grant used to construct the computer cluster where most of these calculations were run and the JPL/Caltech Supercomputer Project for additional computing resources.

${ }^{1}$ For a recent, comprehensive review, see L. G. Christophorou and J. K. Olthoff, J. Phys. Chem. Ref. Data 29, 267 (2000).

${ }^{2}$ H. Cho, R. J. Gulley, and S. J. Buckman, J. Phys. B 33, L309 (2000).

${ }^{3}$ H. Cho, R. J. Gulley, K. W. Trantham, L. J. Uhlmann, C. J. Dedman, and S. J. Buckman, J. Phys. B 33, 3351 (2000).

${ }^{4}$ F. A. Gianturco, R. R. Lucchese, and N. Sanna, J. Chem. Phys. 102, 5743 (1995).

${ }^{5}$ F. A. Gianturco and R. R. Lucchese, J. Chem. Phys. 114, 3429 (2001).

${ }^{6}$ S. Trajmar and A. Chutjian, J. Phys. B 10, 2943 (1977).

${ }^{7}$ K. Takatsuka and V. McKoy, Phys. Rev. A 24, 2473 (1981); 30, 1734 (1984).

${ }^{8}$ M. A. P. Lima, L. M. Brescansin, A. J. R. da Silva, C. Winstead, and V. McKoy, Phys. Rev. A 41, 327 (1990).

${ }^{9}$ C. Winstead and V. McKoy, Adv. At., Mol., Opt. Phys. 36, 183 (1996).

${ }^{10}$ C. Winstead and V. McKoy, Comput. Phys. Commun. 128, 386 (2000).

${ }^{11}$ C. Winstead, Q. Sun, and V. McKoy, J. Chem. Phys. 98, 1105 (1993).
${ }^{12}$ L. Boesten, H. Tanaka, A. K. Kobayashi, M. A. Dillon, and M. Kimura, J. Phys. B 25, 1607 (1992).

${ }^{13}$ A. Mann and F. Linder, J. Phys. B 25, 533 (1992).

${ }^{14}$ A. Mann and F. Linder, J. Phys. B 25, 545 (1992).

${ }^{15}$ R. K. Jones, J. Chem. Phys. 84, 813 (1986).

${ }^{16}$ M. J. Frisch, G. W. Trucks, H. B. Schlegel et al., Gaussian 98, Revision A.5, Gaussian, Inc., Pittsburgh, PA, 1998.

${ }^{17}$ W. J. Hunt and W. A. Goddard, Chem. Phys. Lett. 3, 414 (1969).

${ }^{18}$ M. W. Schmidt, K. K. Baldridge, J. A. Boatz et al., J. Comput. Chem. 14, 1347 (1993).

${ }^{19}$ P. J. Hay, J. Am. Chem. Soc. 99, 1013 (1977).

${ }^{20}$ J. F. Ying, T. A. Daniels, C. P. Mathers, H. Zhu, and K. T. Leung, J. Chem. Phys. 99, 3390 (1993), and references therein.

${ }^{21}$ C. Winstead and V. McKoy, Phys. Rev. A 57, 3589 (1998).

${ }^{22}$ C. W. Bauschlicher, J. Chem. Phys. 72, 880 (1980).

${ }^{23}$ T. N. Rescigno and B. I. Schneider, Phys. Rev. A 45, 2894 (1992), and references therein.

${ }^{24}$ J. L. Dehmer and J. Siegel, J. Chem. Phys. 69, 5205 (1978).

${ }^{25}$ R. Rejoub, D. R. Sieglaff, B. G. Lindsay, and R. F. Stebbings, J. Phys. B 34, 1289 (2001).

${ }^{26}$ W. L. Morgan, Adv. At., Mol., Opt. Phys. 43, 79 (2000).

${ }^{27}$ W. L. Morgan, C. Winstead, and V. McKoy (to be published). 